\title{
Del descubrimiento de la insulina a la cirugía metabólica de la diabetes
}

\begin{abstract}
Rafael Álvarez-Cordero ${ }^{1}$
Uno de los múltiples avances de la medicina, derivado de las investigaciones y trabajos de médicos y cirujanos, es el conocimiento de la diabetes mellitus, sus causas y sus tratamientos. En los últimos 15 años se ha llegado a conocer más de esta enfermedad que en todos los siglos anteriores. Este artículo intenta resumir esta sorprendente historia.
\end{abstract}

La diabetes mellitus es una enfermedad milenaria. Desde 1,500 años antes de nuestra era se conocía su lenta y penosa evolución en un individuo que perdía peso, estaba sediento todo el día, orinaba sin cesar (por eso el nombre de diabetes, paso de orina mencionado por primera vez por Areteo de Capadocia) y moría pronto. Siglos después, en 1673 Thomas Willis añadió el nombre de mellitus (semejante a la miel) por el sabor que tenía la orina de estos enfermos.

Durante siglos no hubo avance en el manejo de esta enfermedad y a finales del siglo XIX el papel del páncreas en el proceso fue conociéndose cada vez más. El célebre doctor Claude Bernard participó en estos estudios y muchos investigadores trataron de desarrollar técnicas para extraer las sustancias del páncreas que pudieran aliviar la diabetes.

Fueron dos investigadores Frederick G. Banting y Charles Best quienes en su laboratorio de Toronto aislaron la sustancia que llamaron isletina (por proceder de los islotes de Langerhans) y en 1922 la aplicaron por primera vez a un joven diabético, Leonard Thompson. Los resultados fueron alentadores y poco después la hija del Gobernador de Nueva York, Elizabeth Huges recibió

\footnotetext{
${ }^{1}$ Fundador y Presidente Honorario del Colegio Mexicano de Cirugía para la Obesidad y Enfermedades Metabólicas (CMCOEM).
}

Correspondencia:

Dr. Rafael Álvarez-Cordero

Correo electrónico: raalvare2009@hotmail.com

Aceptado: 17-08-2016.

Este artículo puede ser consultado en versión completa en http:// www.medigraphic.com/actamedica el tratamiento con un éxito rotundo. Los investigadores publicaron su trabajo, ${ }^{1}$ por el que recibieron el premio Nobel de Fisiología y Medicina en 1923.

El estudio del funcionamiento del páncreas y de las sustancias que podrían aliviar la diabetes mellitus continuó, en 1959 apareció la metformina, en 1958 la sulfonilurea y a partir de entonces se han diseñado nuevos fármacos que permiten que el individuo con diabetes tenga un control adecuado de su enfermedad.

¿Y la cirugía? Nadie consideró que los cirujanos tuvieran algo que hacer frente al paciente diabético y se pasó por alto un interesante artículo publicado por el doctor $\mathbf{F}$. Murray que operó a tres enfermos ulcerosos que además padecían diabetes, les cortó el estómago como entonces se hacía y observó que la diabetes se corrigió en los tres pacientes. $^{2}$

Cuando comenzó la cirugía para la obesidad mórbida, las operaciones realizadas lograban una considerable reducción de peso y junto con ello mejoraban la hipertensión, los problemas respiratorios, las lesiones de huesos, la diabetes y otros achaques de los enfermos. Por mucho tiempo se supuso que eso se debía lógicamente a la reducción de peso y a la disminución de la carga metabólica para el organismo enfermo. A través de varias décadas la cirugía de la obesidad o cirugía bariátrica se ha perfeccionado debido a mejor instrumental, mejores suturas y grapas, a la cirugía laparoscópica en los años 80 y actualmente gracias a la cirugía robótica que cuenta con notables ventajas técnicas, menos complicaciones y a la larga un costo/beneficio importante. El cirujano adiestrado en cirugía robótica puede ver los tejidos del paciente en tercera dimensión con todo detalle como nunca antes los había observado y desde su consola dirige con más exactitud cada movimiento que los brazos robóticos reproducen con toda precisión. La robótica 
incorporada a la cirugía de gran obesidad tiene resultados muy satisfactorios.

Y he aquí que en el año 2007 un médico mexicano el doctor Jesús Argüelles Sarmiento expuso en el Congreso Latinoamericano de la Federación Internacional de Cirugía para la Obesidad IFSO celebrado en Iguazú, Brasil ${ }^{3}$ un trabajo en el que ocho individuos con leve sobrepeso y diabetes mellitus fueron operados, les aplicó una técnica semejante a la que se emplea en los grandes obesos, en todos ellos la diabetes remitió y las cifras de glucosa se normalizaron.

Éste fue el arranque, a la par de estudios de otros investigadores, principalmente de Francesco Rubino, se dio un cambio trascendental en el concepto de diabetes que tomó por sorpresa a los cirujanos, pero más a los internistas y endocrinólogos que desde ese momento han realizado numerosas investigaciones para conocer qué es lo que sucede después de la cirugía en el individuo diabético.

De esta manera se han estudiado los cambios hormonales responsables de este fenómeno y entre las múltiples sustancias analizadas se encuentran diversas incretinas, en especial el péptido semejante al glucagón, (GLP1) el péptido inhibidor gástrico (GIP), así como neuropéptido Y, galanina, melanocortina, GSHR y otros más que de una u otra forma cambian el metabolismo del aparato digestivo, en particular del páncreas, con los resultados que hoy conocemos.

Lo anterior ha llevado a los cirujanos y endocrinólogos a organizar múltiples reuniones científicas, tres congresos mundiales sobre la materia y a publicar sus experiencias clínicas con pacientes moderadamente obesos y los resultados de cuidadosos estudios de laboratorio en animales.

Como consecuencia de estos hallazgos todas las asociaciones y sociedades de cirugía de la obesidad cambiaron su nombre y añadieron el término "metabólico" para señalar que se trata no sólo de bajar de peso, sino cambiar positivamente el metabolismo del enfermo; de tal forma que nuestro colegio se llama Colegio Mexicano de Cirugía para la Obesidad y Enfermedades Metabólicas (CMCOEM).

Al igual que en todos los avances de la medicina es indispensable tomar en cuenta el aspecto ético de nuestras intervenciones y las últimas publicaciones, en especial las dos que aparecieron en la revista Diabetes Care, Órgano de la Asociación Americana de Diabetes, que son de gran trascendencia porque analizan no sólo los resultados fríos, las cifras de peso, glucosa, cambios metabólicos, sino que también se solicita la opinión personal de expertos en relación con la indicación de este tipo de intervenciones quirúrgicas. Se estudiaron a profundidad 51 publicaciones internacionales para conocer tanto los resultados de la cirugía ${ }^{4}$ como la opinión de los grupos de especialistas sobre la intervención. ${ }^{5}$

Así, después de una revisión exhaustiva, los expertos concluyen que la cirugía tiene un beneficio real en la vida del enfermo diabético y la evolución de esta enfermedad, que es preciso estudiar cada caso para decidir, de acuerdo con un algoritmo realizado por consenso y propuesto en la segunda publicación, qué pacientes son candidatos y qué pacientes no lo son.

Lo anteriormente expuesto es de suma importancia porque para muchos problemas de salud, en particular la diabetes, se han sugerido decenas de tratamientos, incluso algunos que ofrecen la curación total de un padecimiento que, hasta el día de hoy, sabemos no tiene una cura y que sólo requiere un control adecuado con alimentación, ejercicio, manejo del estrés, medicamentos y actualmente cirugía.

No se ha dicho la última palabra porque seguramente se llegará al conocimiento total de lo que ocurre en el páncreas normal y en el páncreas de un individuo diabético; no obstante, es posible afirmar que la cirugía desempeña en la actualidad un papel determinante en el manejo de la diabetes mellitus, pues es alentador que los avances técnicos como la cirugía robótica permitan que las intervenciones quirúrgicas tengan mejores resultados, lo que merece celebrarse.

\section{REFERENCIAS}

1. Banting FG, Best $\mathrm{CH}$, Collip JB, Campbell WR, Fletcher AA. Pancreatic extracts in the treatment of diabetes mellitus. Can Med Assoc J. 1922; 12: 141-146.

2. Friedman MN, Sancetta AJ, Magovern GJ. Amelioration of dibetes mellitus after subtotal gastrectomy. Surg Gynec Obst. 1955; 100: 201-204.

3. Arguelles SJ. Efecto de la cirugía bariátrica en la evolución de la diabetes en un grupo de pacientes con sobrepeso. Proceedings del Congreso Latinoamericano IFSO Iguazú, Septiembre 2007; p. 21.

4. Cefalu W, Rubino F, Cummings DE. Metabolic surgery for type 2 diabetes, changing the landscape of diabetes care. Diabetes Care 2016; 39: 857-860.

5. Rubino F, Nathan DM, Eckel RH, Schauer PR, Alberti KG, Zimmet PZ et al. Metabolic surgery in the treatment algorithm for type 2 diabetes; a joint statement by international diabetes organizations. Diabetes Care. 2016; 39: 861-877. 